\title{
Near-infrared imaging of RAFGL7009S ${ }^{\star}$
}

\author{
A. Zavagno ${ }^{1}$, L. Deharveng ${ }^{1}$, D. Nadeau ${ }^{2}$, and J. Caplan ${ }^{1}$ \\ 1 Observatoire de Marseille, 2 place Le Verrier, 13248 Marseille Cedex 4, France \\ 2 Observatoire du Mont Mégantic et Département de Physique, Université de Montréal, CP 6128, Succ. Centre-ville, Montréal, \\ QC, Canada H3C 3J7 \\ e-mail: nadeaud@ere.umontreal.ca
}

Received 13 March 2002 / Accepted 29 July 2002

\begin{abstract}
RAFGL7009S is a deeply embedded massive young stellar objet (YSO) showing strong ice and saturated silicate absorption features in the mid infrared. It is associated with the ultracompact $\mathrm{H}$ II region $\mathrm{G} 25.65+1.05$, which may be excited by a B1V star. We have obtained $J H K$ images of a 1' field centred on this YSO. In $K$ we detect a non-resolved object coinciding with the radio continuum emission peak. Considering the high extinction towards this source $\left(A_{V} \geq 100 \mathrm{mag}\right)$, the observed $K$ emission is more than $7 \mathrm{mag}$ in excess of that expected for a B1V star. We suggest that this emission is mainly due to scattering of the central zone emission, as recently found for a sample of embedded massive YSOs associated with $3.1 \mu \mathrm{m} \mathrm{H}_{2} \mathrm{O}$ ice absorption (Ishii et al. 2002). We estimate the YSO's age to be $2 \times 10^{4}$ years. The outflow, the methanol maser emission and the strong ice absorption features suggest the presence of a dense medium very close to the star, possibly a disk.
\end{abstract}

Key words. stars: formation - stars: pre-main sequence - stars: imaging - stars: individual: RAFGL7009S

\section{Introduction}

During the early stages of their formation, YSOs are deeply embedded in their parental cocoons of gas and dust, and are thus subject to high extinction. If it is massive, the young star ionizes the surrounding medium, creating an ultracompact $\mathrm{H}_{\text {II }}$ region. The UV and visible photons leaking from the $\mathrm{H}$ II region heat the dust. The hot dust, located close to the star, reradiates this energy at near- and mid-IR wavelengths. The large cold envelope surrounding this ensemble would normaly prevent this radiation from escaping, thus prohibiting direct observation of an embedded young massive star in the near and mid-IR at the beginning of its formation. Nevertheless, many highly embedded YSOs do have near-IR counterparts despite their high visual extinctions estimated from the silicate absorption feature at $9.7 \mu \mathrm{m}$ (Faison et al. 1998; Walsh et al. 1999; van der Tak et al. 2000). These highly embedded sources are detected in $K$ but have no $J$ and $H$ counterparts. A faint $K$ emission is observed, and the emission becomes brighter at longer wavelengths. These objects are very red with $K-L>5$ (e.g. NIRS 3 in Sh 255-2, Itoh et al. 2001).

We present here the case of RAFGL7009S. This infrared source (also known as IRAS 18316-0602) is a typical example of a massive and deeply embedded object, and the

Send offprint requests to: A. Zavagno,

e-mail: zavagno@observatoire.cnrs-mrs.fr

* Table 1 is only available in electronic form at the CDS via anonymous ftp to cdsarc.u-strasbg.fr (130.79.128.5) or via http://cdsweb.u-strasbg.fr/cgi-bin/qcat?J/A+A/394/225 textbook case for the study of ices towards embedded massive young stars (d'Hendecourt et al. 1996; Dartois et al. 2000). This source is associated with the ultracompact $\mathrm{H}$ II region G25.65+1.05. In Sect. 2 we describe the RAFGL7009S source and its environment. Section 3 presents near-IR observations of this source; an unresolved $K$ object is observed in its direction. In Sect. 4 we discuss the possible origin of this emission. Conclusions are drawn in Sect. 5.

\section{The RAFGL7009S environment}

The kinematic distance of this source, based on the radial velocity of the molecular material $\left(V_{\mathrm{LSR}}(\mathrm{CO})=41.5 \mathrm{~km} \mathrm{~s}^{-1}\right.$, Shepherd \& Churchwell 1996; Bronfman et al. 1996) and on the Galactic rotation curve of Brand \& Blitz (1993), is either 3.0 or $12.3 \mathrm{kpc}$. In the following we adopt the near distance of $3.0 \mathrm{kpc}$ favoured by our photometric results (see Sect. 3). Also, as stressed by McCutcheon et al. (1991), the use of the far distance implies an unrealistically high luminosity.

RAFGL7009S is associated with all the usual tracers of massive star formation: high IR luminosity, an ultracompact radio continuum source, energetic outflow(s) and masers. The associated radio continuum source, G25.65+1.05, has been observed at $3.6 \mathrm{~cm}$ (Kurtz et al. 1994), at $6 \mathrm{~cm}$ (McCutcheon et al. 1995) and at $3.5 \mathrm{~cm}$ (Walsh et al. 1998). Its high emission measure $\left(\geq 44 \times 10^{5} \mathrm{pc} \mathrm{cm}^{-6}\right)$ and high electron density $\left(\geq 15 \times 10^{3} \mathrm{~cm}^{-3}\right.$ ) derived from the $6 \mathrm{~cm}$ observations show that this source is most probably an ultracompact $\mathrm{H}$ II region (see Sect. 4.1). The resolved (diameter 3") $3.6 \mathrm{~cm}$ radio source 


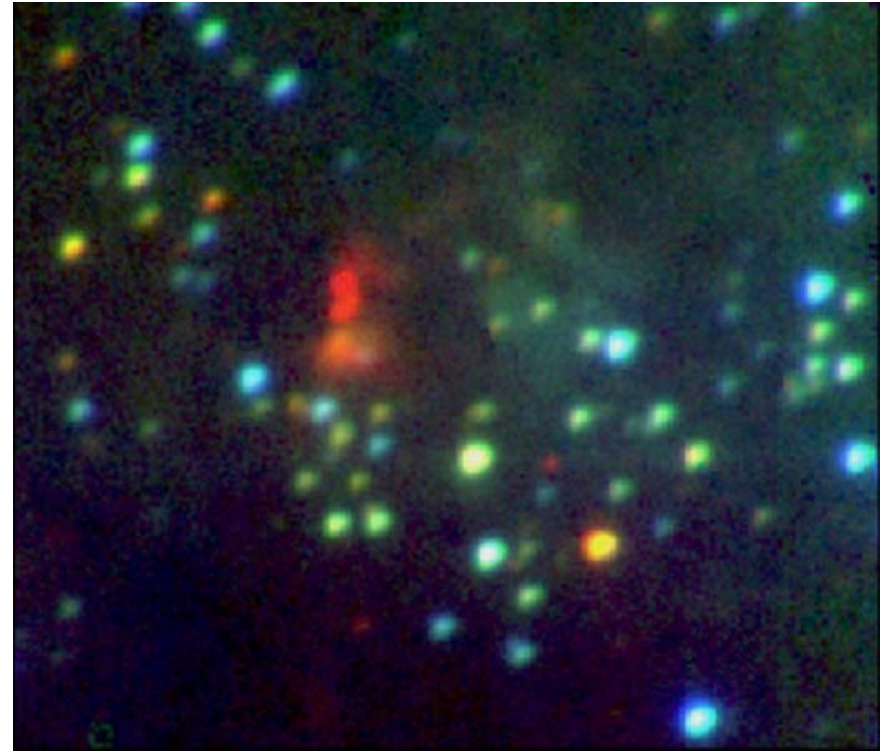

Fig. 1. Colour composite image of $\mathrm{G} 25.65+1.05$ ( $J$ is blue, $H$ is green and $K$ is red). North is up and east is left. The field is $47^{\prime \prime} \times 41^{\prime \prime}$.

is located at $\alpha=18^{\mathrm{h}} 31^{\mathrm{m}} 40^{\mathrm{s}} 15, \delta=-06^{\circ} 02^{\prime} 06^{\prime \prime} 3$ (B1950). It coincides with the IR source IRAS 18316-0602 whose luminosity is $2.87 \times 10^{4} L_{\odot}$ (McCutcheon et al. 1995). A highly energetic molecular outflow is centred on G25.65+1.05 (Shepherd \& Churchwell 1996). An east-west extension of the $\mathrm{CH}_{3} \mathrm{CN}$ emission (Dartois et al. 2000) has been attributed to the outflow. The map of the high velocity component of the outflow (see Fig. 5b in Shepherd \& Churchwell 1996) suggests the presence of two bipolar flows. Multiple outflows have been observed in a few other YSO regions (Henning et al. 2000; Goetz et al. 1998) and are attributed to the presence of several massive stars, each driving its own outflow. This may be an indication that the central source of RAFGL7009S is not single. However, it is impossible with the present data to constrain the spectral type of a possible companion (Sect. 4.2). A bright water maser has been observed in the direction of IRAS 18316-0602 by Palla et al. (1991). Four methanol maser spots have been observed by Walsh et al. (1998). Those spots are distributed in a chain, 0 '. 5 to 1 '.5 southwards of the radio source, and may be associated with the outflow (Lee et al. 2001) or with a disk (de Buizer et al. 2001). Flux measurements have been obtained at $350 \mu \mathrm{m}$ (Hunter et al. 2000), $450 \mu \mathrm{m}, 800 \mu \mathrm{m}$ and $1100 \mu \mathrm{m}$ (McCutcheon et al. 1995). The $10-1100 \mu \mathrm{m}$ spectral energy distribution can be fitted by a two-component spherical distribution, a small warm component of diameter $5^{\prime \prime}(0.073 \mathrm{pc})$ with a temperature of $123 \mathrm{~K}$ and a cold component of diameter $20^{\prime \prime}$ $(0.30 \mathrm{pc})$ and a temperature of $35 \mathrm{~K}$ (McCutcheon et al. 1995; see also Hunter et al. 2000). These results agree with the typical values derived by van der Tak et al. (2000) for a sample of fourteen massive, deeply embedded YSOs. The emission of the cold envelope associated with RAFGL7009S has also been observed at $2.7 \mathrm{~mm}$ (Dartois et al. 2000).

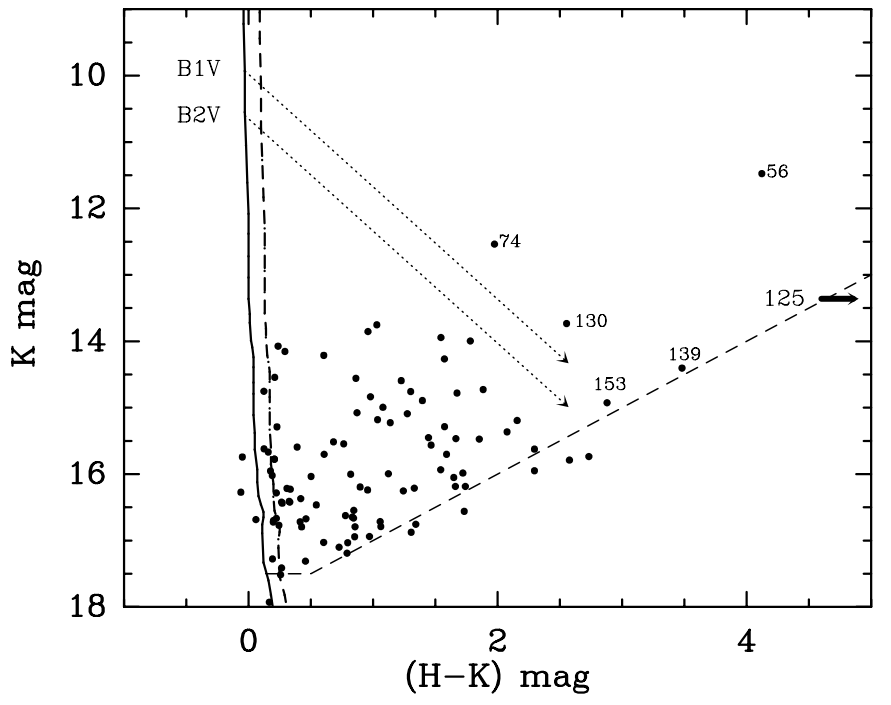

Fig. 2. The $K$ versus $H-K$ diagram. The main sequence is from Vacca et al. (1996) for O stars and from Schmidt-Kaler (1982) for later spectral types; the solid line corresponds to the main-sequence with zero extinction and the dashed line corresponds to 2 mag of visual extinction. Two reddening lines (dotted) originating from a B1V and a B2V star correspond to a visual extinction of $40 \mathrm{mag}$ and the standard interstellar reddening law of Mathis (1990) with $R_{V}=3.1$. The dashed lines show our detection limits.

\section{Near-IR observations}

The observations were obtained with the Montreal Near-IR Camera (MONICA, Nadeau et al. 1994) mounted at the F/8 Cassegrain focus of the 3.6-m Canada-France-Hawaii Telescope on 1996 July 1 UT. Frames were obtained through the $J, H$ and $K$ broad-band filters with total integration times of $840 \mathrm{~s}, 160 \mathrm{~s}$ and $100 \mathrm{~s}$. Details about the instrumentation, the calibration and the data analysis can be found in Deharveng et al. (1999). The $F W H M$ of the $K$ PSF is 0!'7. Figure 1 presents a composite colour image of the region. The colour differences of the stars are mainly due to differences in their reddening.

Table 1 (available only in electronic form) contains $J H K$ magnitudes and astrometry for 174 stars detected in the $63^{\prime \prime} \times 70^{\prime \prime}$ field around G25.65+1.05. The unresolved bright red object (detected only in $K$ ) is object 125 in Table 1 . Its $K$ magnitude is 13.36 . Its non-detection in $J$ indicates that $J-K>6.44$ mag. Its B1950 coordinates are $18^{\mathrm{h}} 31^{\mathrm{m}} 40 \mathrm{~s} 15$, $-06^{\circ} 02^{\prime} 6.2^{\prime \prime}$ and it coincides with the $3.6 \mathrm{~cm}$ radio emission peak (Kurtz et al. 1994). We discuss this point in Sect. 4.

The $K$ versus $H-K$ diagram is presented in Fig. 2. The stars discussed in the text are identified in Fig. 3. This diagram shows the following: i) Adopting the far distance of $12.3 \mathrm{kpc}$ would lead to 3.1 mag brighter absolute magnitudes for all the stars. Stars that luminous would ionize their own $\mathrm{H}$ II regions, but these are not detected. ii) A number of stars are shifted from the main sequence by some 2 mag of visual extinction. These are probably foreground stars. iii) Among the stars detected in $H$ and $K$, a few stars display high extinction, appearing as red or orange in Fig. 1. These are stars 56, 74, 130, 139, and 153, with $A_{V}$ in the range $30-50 \mathrm{mag}$. Star 56 is very red and bright 


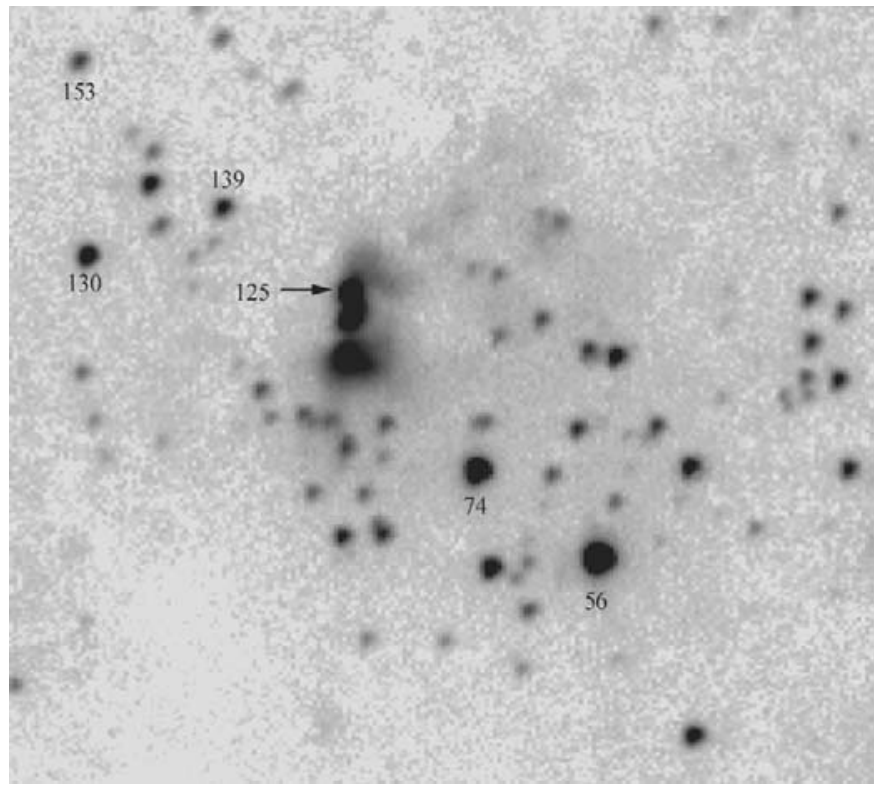

Fig. 3. $K$ frame. The stars discussed in the text are identified by their numbers in Table 1 .

in $K$ but, due to its non detection in $J$, we have no information about a possible near-IR excess. Star 74 has no near-IR excess and could be a B0V star. However no continuum emission is associated with these stars in the NVSS survey at $21 \mathrm{~cm}$ (Condon et al. 1998), down to a flux density of $2.5 \mathrm{mJy}$. The associated $\mathrm{H}$ II regions, if any, may be optically thick at $21 \mathrm{~cm}$, or these stars may be massive YSOs with high accretion rates that prevent the formation of an H II region (Walmsley 1995).

Nebulous emission is observed in $K$ (Fig. 1). Two small sources are observed to the south and a fainter more extended nebulosity is located to the north-west. The first of the southern sources is 1 ." 3 from the central object 125 and has a $K$ brightness of $13.7 \mathrm{mag} /\left({ }^{\prime \prime}\right)^{2}$ with a PSF-corrected $F W H M$ of 0 ' 9 ; the second is 3".6 from the central object and is more extended and fainter, with a $F W H M$ of 2 '. 4 and $13.9 \mathrm{mag} /\left({ }^{\prime \prime}\right)^{2}$. The north-west nebulosity has a lower surface brightness and is arc-shaped, convex toward object 125 . The outer velocity contour of the red wing of the CO outflow (see Fig. 5b in Shepherd \& Churchwell 1996) encloses this north-western nebulous emission. This emission observed in the $K$ band is possibly shocked $\mathrm{H}_{2}$ emission at $2.12 \mu \mathrm{m}$ due to the propagation of the outflow into the surrounding medium (cf. Itoh et al. 2001 and Lee et al. 2001). The main blue wing of the outflow, located to the east of the central source, is not detected in our $K$ image. This eastern zone seems to be highly attenuated by the dense cold envelope.

If the $3.6 \mathrm{~cm}$ emitting region is optically thin at this wavelength, its contribution to the millimetre emission is negligible. Therefore the observed $2.7 \mathrm{~mm}$ emission (see Fig. 3 in Dartois et al. 2000) must come from the cold dust envelope that surrounds the star. Figure 1 shows that no star is observed inside a $15^{\prime \prime}$ diametre zone centred on object 125 , a zone that corresponds to the size of the surrounding envelope. The nondetection, both in $\mathrm{K}$ and in $\mathrm{CH}_{3} \mathrm{CN}$, of emission associated

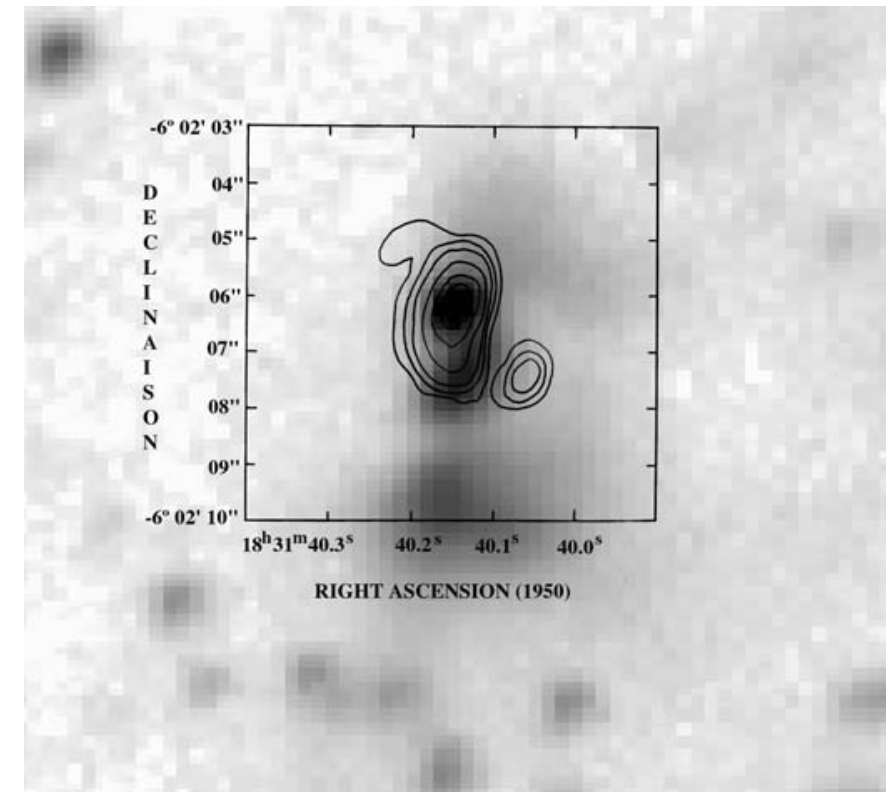

Fig. 4. The $3.6 \mathrm{~cm}$ radio continuum map of G25.65+1.05 (Kurtz et al. $1994)$ superimposed on the $K$ frame of RAFGL7009S. Note the coincidence between the radio emission peak and the central $K$ unresolved object.

with the blue wing of the outflow suggests that the eastern part of the envelope is denser (see also Dartois et al. 2000).

\section{Discussion}

Figure 4 presents the $3.6 \mathrm{~cm}$ radio map of G25.65+1.05 (Kurtz et al. 1994) superimposed on our $K$ frame. Object 125 coincides with the radio emission peak.

\subsection{The nature of the radio source $G 25.65+1.05$}

The measured flux densities of $3.8 \mathrm{mJy}$ at $3.6 \mathrm{~cm}$ (Kurtz et al. 1994) and $2.7 \mathrm{mJy}$ at $6 \mathrm{~cm}$ (McCutcheon et al. 1995), together with the upper limit of $2.5 \mathrm{mJy}$ imposed by the non-detection of the source in the NVSS survey at $21 \mathrm{~cm}$, make the source spectrum compatible with that of an ionized stellar wind $\left(S_{v} \propto v^{0.6}\right.$; Simon et al. 1983). However, in this case, the radio source should not be resolved - but it is (Kurtz et al. 1994). These flux measurements are also compatible with the thermal spectrum of an $\mathrm{H}_{\mathrm{II}}$ region, assuming that the source is optically thick at $21 \mathrm{~cm}$ which is possible given its high emission measure at $6 \mathrm{~cm}$ (McCutcheon et al. 1995). In this case, G25.65+1.05 would be an ultracompact $\mathrm{H}$ II region. In the following, we assume that this is the case.

\subsection{The G25.65+1.05 exciting star}

Assuming that G25.65+1.05 is optically thin at $3.6 \mathrm{~cm}$ and that a single main-sequence star dominates its ionization, the spectral type of the exciting star can be estimated from its radio flux density. (For the ionizing stars of ultracompact H II regions, Hanson et al. 2001 find a good agreement between the spectral type estimated from the radio continuum flux and that 
determined from near-IR spectra.) We have used Eq. (1) of Simpson \& Rubin (1990) to determine $N_{\text {Lyc }}$, the number of Lyman continuum photons emitted per second by the star. Assuming a distance of $3.0 \mathrm{kpc}$, no $\mathrm{He}^{+}$, and an electron temperature of $7440 \mathrm{~K}$ (Afflerbach et al. 1996), the flux density of $3.8 \mathrm{mJy}$ (Kurtz et al. 1994) gives $\log N_{\text {Lyc }}=45.56$. According to Panagia (1973) this corresponds to a B1V exciting star. However, the relation between the spectral type and the Lyman continuum flux is highly uncertain; e.g. Vacca et al. (1996) associate a B1V star with a Lyman continuum flux six times higher than Panagia's (1973). Under these conditions we are unable to ascertain whether or not the exiting star is multiple.

\subsection{Origin of the observed $\mathrm{K}$ emission}

The $J$ magnitude of an unreddened B1V star lying at $3.0 \mathrm{kpc}$ is 9.9 mag. Assuming that the central source is a B1V star, its non-detection within our $J$ detection limit of about 19.8 mag gives $A_{J}>10 \mathrm{mag}$ and thus $A_{V}>36 \mathrm{mag}$ for a standard extinction law (Mathis 1990). The ISO spectra of this source clearly indicate that the true extinction is much greater. In particular, various ice absorption features (d'Hendecourt et al. 1996) and deep, saturated silicate absorption at $9.6 \mu \mathrm{m}$ (Demyk et al. 1999) indicate that this source is affected by more than $75 \mathrm{mag}$ of visual extinction (Dartois et al. 1999). Note that this is a lower limit (cf. the similar case of Cep A East, van den Ancker et al. 2000). Dartois et al. (2000) have determined a mean molecular hydrogen density of $10^{6} \mathrm{~cm}^{-3}$ within a sphere of $10-15^{\prime \prime}$ diameter assumed to contain all the associated molecular material. This corresponds to a column density of molecular hydrogen of $4.5 \times 10^{23} \mathrm{~cm}^{-2}$. Using the relation given by Ryter (1996) between the visual extinction and the hydrogen column density, we find a value of $A_{V}=205 \mathrm{mag}$. Assuming that the exciting star lies at the centre of the envelope, it is affected by 102 mag of visual extinction, thus $A_{K}=10.9$ mag. The $K$ magnitude of a B1V star at $3 \mathrm{kpc}$ with an extinction of $A_{K}=10.9 \mathrm{mag}$ is 20.8 . Thus, object 125 with a $K$ magnitude of 13.36 cannot be the exciting star itself.

Assuming optically thin thermal radio emission, we extrapolated the $3.6 \mathrm{~cm}$ flux and found the free-free contribution of the ionized gas at $K$ to be about $1.4 \mathrm{mJy}$ (without extinction). This emission is produced at the centre of the envelope and is affected by the same high extinction as the star. Thus the freefree contribution at $K$ corresponds to $27.18 \mathrm{mag}$ and cannot account for the observed $K$ emission of object 125 .

Thermal emission from hot dust may be at the origin of the observed emission. To radiate significantly at $K$, the dust temperature must be near the sublimation temperature $(\simeq 1200 \mathrm{~K})$. For early B type stars, a dust temperature of $1000 \mathrm{~K}$ is reached at a distance of about 50 AU (Osorio et al. 1999). This corresponds to 15 mas for a source located at $3 \mathrm{kpc}$. However, this emission suffers the same extinction as the star does and cannot be directly detected.

The possible contributors to the $K$ emission are: the star, the hot dust located close to the star, and the free-free emission of the ionized gas. The hot dust emission probably dominates the $K$ flux (see also Lumsden \& Puxley 1996). However, we have shown that all these emissions suffer a high extinction and cannot be directly observed. Nevertheless $K$ emission is often observed associated with deeply embedded massive YSOs. To resolve this dilemma, we propose that the radiation emitted by the central zone is scattered and escapes through cavities carved out by the outflows.

\subsection{RAFGL7009S is not a unique case}

The spectral energy distribution of RAFGL7009S (see Fig. 1 in Dartois et al. 2000) peaks around $120 \mu \mathrm{m}$, as is typical of embedded massive YSOs (van der Tak et al. 2000). Our measured $K$ flux of $3.0 \mathrm{mJy}$ agrees well with the ISO-SWS measurements (see Fig. 1 in Dartois et al. 1998). A ground-based spectrum in the 3.42-4.06 $\mu \mathrm{m}$ range (Dartois et al. 1999) also agrees with the ISO data. Using these data we derive $L$ and $M$ magnitudes of 7.90 and 2.44, respectively, and hence a $K-L$ of 5.46. This is typical of highly embedded sources (Walsh et al. 1999). Note that the source NIRS 3 in Sh 255-2 exhibits very similar properties (Itoh et al. 2001); it ionizes an ultracompact H II region and has very red colours. Walsh et al. (1998) give other examples of such very red sources towards regions of massive star formation. These sources are not detected in $J$ and $H$ but are detected in $K$ and at longer wavelengths. The measured $K$ emission in these sources varies from $1 \mathrm{mJy}$ to 1 Jy (van der Tak et al. 2000).

\subsection{Possible presence of a disk?}

Polarimetric imaging of deeply embedded sources (Yao et al. 2000 and references therein) reveal "polarization disks" - regions of elliptically- rather than circularly-symmetric polarization vectors - towards the embedded sources, indicative of dense circumstellar disks. The presence of a disk around the massive protostar AFGL 2136 IRS1 has also been suggested by 2-4 $\mu \mathrm{m}$ imaging (Kastner \& Weintraub 1996). Mid-IR imaging of deeply embedded massive stars also suggest that the absorbing material is highly localised and may be in a disk (Kraemer et al. 2001).

Some exciting stars of compact $\mathrm{H}_{\text {II }}$ regions exhibit a $K$ overluminosity and near-IR excess attributed to the presence of a disk (see, for example, the case of Sh 138, Deharveng et al. 1999). Indeed, young massive stars in M 17 that show evidence for a disk, through near-IR CO band-head emission at $2.3 \mu \mathrm{m}$, are overluminous in $K$ (Hanson et al. 1997). The presence of a hot circumstellar disk towards the cometary $\mathrm{H}_{\text {II }}$ region $\mathrm{G} 34.3+0.2 \mathrm{C}$ is also revealed by a $K$ and $L$ emission excess and a broken toroid-like structure, less than $1^{\prime \prime}$ in size, observed in both filters (Gaume et al. 2000).

Two observational facts suggest the presence of a circumstellar disk in RAFGL7009S: i) Methanol maser emissions are observed towards the compact $\mathrm{H}_{\text {II }}$ region, suggesting a dense medium (Walsh et al. 1998). ii) The maser components towards this region are aligned north-south, perpendicular to the flow axis. Strong absorption features from various ices are also observed in this source (d'Hendecourt et al. 1996), especially a saturated absorption feature due to water ice, observed at 
$3.08 \mu \mathrm{m}$. Such a deep absorption has also been observed in AFGL 2136 IRS1 and may be located, in part, in a disk, according to Kastner \& Weintraub (1996).

\subsection{The age of RAFGL7009S}

The dynamical timescale of the outflow is $1.9 \times 10^{4}$ years (Shepherd \& Churchwell 1996). A dynamical age can also be estimated for the $\mathrm{H}$ II region, based on its expansion and present-day size. According to Spitzer (1978), an H II region which develops in a medium of density $10^{6} \mathrm{~cm}^{-3}$ (Dartois et al. 2000) reaches a radius of 1 .' 5 (the present radius of the radio source (Kurtz et al. 1994), in $1.8 \times 10^{4}$ years. This age, in good agreement with the dynamical age of the outflow, confirms that RAFGL7009S is young.

\section{Conclusions}

$J H K$ imaging towards the embedded massive YSO RAFGL7009S reveals an unresolved (FWHM $<0$ ! $)$ $K$ object that coincides with the peak emission of the ultracompact radio source $\mathrm{G} 25.65+1.05$ and with the peak of millimetre dust emission. Several indicators such as its high luminosity, its association with an ultracompact $\mathrm{H}_{\text {II }}$ region, its high extinction, its spectral energy distribution showing a high IR excess, the highly energetic outflow (possibly multiple) and maser emissions point to RAFGL7009S being a young embedded massive star. The dynamical timescale of the outflow, together with the dynamical age of the ultracompact radio source, also suggest a young source, about $2 \times 10^{4}$ years old. The high inferred extinction towards this source $\left(A_{V} \geq 100 \mathrm{mag}\right)$ indicates that the observed $K$ emission is likely to be due to scattering of the central source emission (star + hot dust + free-free) passing through the outflow cavities. The possible presence of a disk, suggested by many independant observational aspects, should be investigated by near-IR polarimetry (cf. Yao et al. 2000) and $K$-band spectroscopy (cf. Hanson et al. 1997).

Acknowledgements. Neil Commons, the student who originally worked on the data reduction, is warmly thanked. Stan Kurtz and Andrew Walsh are thanked for providing information on the source. This work has made use of the Simbad bibliographic service.

\section{References}

Afflerbach, A., Churchwell, E., Acord, J. M., et al. 1996, ApJS, 106, 423

de Buizer, J. M., Piña, R. K., \& Telesco, C. M. 2000, ApJS, 130, 437

Brand, J., \& Blitz, L. 1993, A\&A, 275, 67

Bronfman, L., Nyman, L.-A., \& May, J. 1996, A\&AS, 115, 81

Condon, J. J., Cotton, W. D., Greisen, E. W., et al. 1998, AJ, 115, 1693

Dartois, E., Cox, P., Roelfsema, P. R., et al. 1998, A\&A, 338, L21

Dartois, E., Schutte, W., Geballe, T. R., et al. 1999, A\&A, 342, L32
Dartois, E., Gerin, M., \& d'Hendecourt, L. 2000, A\&A, 361, 1095

Deharveng, L., Zavagno, A., Nadeau, D., Caplan, J., \& Petit, M. 1999, A\&A, 344, 943

Demyk, K., Jones, A. P., Dartois, E., Cox, P., \& d'Hendecourt, L. 1999, A\&A, 349, 267

Faison, M., Churchwell, E., Hofner, P., et al. 1998, ApJ, 500, 280

Gaume, R. A., Lindqvist, M., Black, J. H., et al. 2000, BAAS, 197, 4307

Goetz, J. A., Pipher, J. L., Forrest, W. J., et al. 1998, ApJ, 504, 359

Hanson, M. M., Howarth, I. D., \& Conti, P. S. 1997, ApJ, 489, 698

Hanson, M. M., Luhman, K. L., \& Rieke, G. H. 2002, ApJS, 138, 35

d'Hendecourt, L., Jourdain de Muizon, M., Dartois, E., et al. 1996, A\&A, 315, L365

Henning, Th., Schreyer, K., Launhardt, R., \& Burkert, A. 2000, A\&A, 353, 211

Hunter, T. R., Churchwell, E., Watson, C., et al. 2000, AJ, 119, 2711

Ishii, M., Hirao, T., Nagashima, C., et al. 2002, AJ, in press

Itoh, Y., Tamura, M., Suto, H., et al. 2000, PASJ, 53, 495

Kastner, J. H., \& Weintraub, D. A. 1996, ApJ, 466, L103

Kurtz, S., Churchwell, E., \& Wood, D. O. S. 1994, ApJS, 91, 659

Kraemer, K. E., Jackson, J. M., Deutsch, L. K., et al. 2001, ApJ, 561, 282

Lee, J.-K., Walsh, A. J., Burton, M. G., \& Ashley, M. C. B. 2001, MNRAS, 324, 1102

Lumsden, S. L., \& Puxley, P. J. 1996, MNRAS, 281, 493

Mathis, J. S. 1990, ARA\&A, 28, 37

McCutcheon, W. H., Dewdney, P. E., Purton, C. R., \& Sato, T. 1991, AJ, 101, 1435

McCutcheon, W. H., Sato, T. O., Purton, C. R., Mathews, H. E., \& Dewdney, P. E. 1995, AJ, 110, 1762

Nadeau, D., Murphy, D. C., Doyon, R., \& Rolands, N. 1994, PASP, 106, 909

Osorio, M., Lizano, S., \& D’Alessio, P. 1999, ApJ, 525, 808

Palla, F., Brand, J., Cesaroni, R., Comoretto, G., \& Felli, M. 1991, A\&A, 246, 249

Panagia, N. 1973, AJ, 78, 929

Ryter, C. E. 1996, ApSS, 236, 285

Schmidt-Kaler, T. 1982, in Landolt-Bornstein, Group IV, vol. 2, ed. K. Schaifers, \& H. H. Voigt (Springer-Verlag, Berlin), 1

Shepherd, D. S., \& Churchwell, E. 1996, ApJ, 457, 267

Simon, M., Felli, M., Massi, M., Cassar, L., \& Fischer, J. 1983, ApJ, 266, 623

Simpson, J. P., \& Rubin, R. H. 1990, ApJ, 354, 165

Spitzer, L., 1978, in Physical Processes in the Interstellar Medium, Chap. 12 (Wiley-Interscience publication, New York)

Vacca, W. D., Garmany, C. D., \& Shull, J. M. 1996, ApJ, 460, 914

van den Ancker, M., Tielens, A. G. G. M., \& Wesselius, P. R. 2000, A\&A, 358, 1035

van der Tak, F. F. S., van Dishoeck, E., Evans II, N. J., \& Blake, G. A. 2000, ApJ, 537, 283

Walsh, A. J., Burton, M. G., Hyland, A. R., \& Robinson, G. 1998, MNRAS, 301, 640

Walsh, A. J., Burton, M. G., Hyland, A. R., \& Robinson, G. 1999, MNRAS, 309, 905

Walmsley, M. 1995, Rev. Mex. Astron. Astrofis. Ser. Conf., 1, 137

Yao, Y., Ishii, M., Nagata, T., Nakaya, H., \& Sato, S. 2000, ApJ, 542, 392 